\title{
Hard-core deconfinement and soft-surface delocalization from nuclear to quark matter
}

\author{
Kenji Fukushima, ${ }^{1, *}$ Toru Kojo, ${ }^{2, \dagger}$ and Wolfram Weise ${ }^{3,+}$ \\ ${ }^{1}$ Department of Physics, The University of Tokyo, 7-3-1 Hongo, Bunkyo-ku, Tokyo 113-0033, Japan \\ ${ }^{2}$ Key Laboratory of Quark and Lepton Physics (MOE) and Institute of Particle Physics, \\ Central China Normal University, Wuhan 430079, China \\ ${ }^{3}$ Physics Department, Technical University of Munich, 85748 Garching, Germany
}

(Received 14 September 2020; accepted 28 October 2020; published 16 November 2020)

\begin{abstract}
We propose a conceptual distinction between hard and soft realizations of deconfinement from nuclear to quark matter. In the high density region of hard deconfinement the repulsive hard cores of baryons overlap each other and bulk thermodynamics is dominated by the core properties that can be experimentally accessed in high-energy scattering experiments. We find that the equation of state estimated from a single baryon core is fairly consistent with those empirically known from neutron star phenomenology. We next discuss a novel concept of soft deconfinement, characterized by quantum percolation of quark wave functions, at densities lower than the threshold for hard deconfinement. We make a brief review of quantum percolation in the context of nuclear and quark matter and illustrate a possible scenario of quark deconfinement at high baryon densities.
\end{abstract}

DOI: 10.1103/PhysRevD.102.096017

\section{INTRODUCTION}

Microscopic mechanisms of the deconfinement phenomenon from nuclear to quark matter are still veiled in mystery. There are countless numbers of theoretical attempts since the first speculation on quark matter in Refs. [1,2], but we have not established any convincing picture to understand deconfinement along the baryon density axis (see Refs. [3-6] for reviews on the phase diagram and various conceivable scenarios at high density, see also Ref. [7] for a review on experimental efforts to reveal the phase diagram). To gain a theoretical understanding, solving the first-principles theory, i.e., quantum chromodynamics $(\mathrm{QCD})$ is not necessarily a unique route as it is severely hindered by the notorious sign problem $[8,9]$. In this work we are proposing a novel viewpoint to make a conceptual differentiation between hard and soft realizations of deconfinement and interpret quark matter based on a condensed-matter physics analogue.

To make our proposal clearer, it is instructive to start with our understanding of deconfinement in a different

\footnotetext{
fuku@nt.phys.s.u-tokyo.ac.jp

kojo.toru@gmail.com

*weise@tum.de
}

Published by the American Physical Society under the terms of the Creative Commons Attribution 4.0 International license. Further distribution of this work must maintain attribution to the author(s) and the published article's title, journal citation, and DOI. Funded by SCOAP . environment, at high temperature $T$ and low baryon density $\rho$, rather than high density and low temperature of our current interest. The great advantage in such an environment at high $T$ and low $\rho$ is that the lattice-QCD simulations provide detailed quantitative information on deconfinement along the $T$-axis as long as $\rho$ is small. It is known that deconfinement from hadronic matter to a quarkgluon plasma (QGP) occurs continuously at physical quark masses. In other words, there is no genuine phase transition in a strict sense: physical degrees of freedom should be smoothly connected from hadronic to QGP matter. We note that there are several approximate and phenomenological measures of quark deconfinement by means of fluctuations $[10,11]$. Especially the quartic to quadratic ratio of the baryon number fluctuation was first considered in this way [12]. The lattice-QCD results including these fluctuations gave us a guiding principle, but it is still nontrivial whether gauge-invariant thermodynamic quantities can diagnose contents of physical degrees of freedom. They should be hadrons at low $T$ and change to quarks and gluons at high enough $T$, but these degrees of freedom should be converted in an intermediate region without a phase transition.

The success of the hadron resonance gas (HRG) model has been a milestone for studies on deconfinement [13-18]. Actually, in the large- $N_{\mathrm{c}}$ limit (where $N_{\mathrm{c}}$ denotes the number of colors), mesons are noninteracting particles and the deconfinement phenomenon of meson-dominated matter is essentially a Hagedorn transition of mesons [19-21], leading to blow-up behavior of thermodynamic quantities. Later on, it has been demonstrated that the 
excluded volume effect would tame singular behavior of thermodynamic quantities [22], which indicates that such a transient state from hadronic matter to the QGP could be approximated well by interacting mesons. This suggests that interhadronic interactions are gradually taken over by partonic degrees of freedom, i.e., quarks and gluons. Here, let us emphasize two properties of high- $T$ deconfinement:

(1) The fact that there is no sharp phase transition allows for significant overlaps of the hadronic and the partonic regimes. The duality in thermodynamics between interacting hadrons and partons holds at overlapping densities. The Polyakov loop is only an approximate order parameter and there is no strict order parameter for deconfinement in the presence of dynamical quarks. One may think that the color conductivity (which has been computed in weakly coupled theories $[23,24]$ ) might play the role of an order parameter, but its behavior is expected to be smooth similarly to the Polyakov loop.

(2) Although there is no clear-cut separation, if thermodynamic quantities are dominated by hadrons (or partons), it would be reasonable to call such a state the hadronic phase (or the QGP, respectively). In the hadronic phase the color-singletness should be imposed locally, but at high enough $T$ more and more multiple interactions would be involved and gradual deconfinement can be possible effectively even under the condition of color neutrality.

One might think that the intuitive understanding based on the HRG model could apply to the high density matter as well, but this expectation would not work straightforwardly. The crucial difference is manifest especially in the large- $N_{\mathrm{c}}$ limit: nucleons are heavy and their kinetic energy is suppressed by $N_{\mathrm{c}}$, while their interaction energy is enhanced as $\mathcal{O}\left(N_{\mathrm{c}}\right)$ [25]. Given such strong interactions, it is far from trivial whether nucleons are really the relevant degrees of freedom or not. In other words, we must revise our notion of deconfinement due to interaction effects when dense baryonic matter is concerned.

The invention of quarkyonic matter [26] has invoked a lot of theoretical arguments along these lines. Quarkyonic matter refers to baryonic matter whose pressure is $\mathcal{O}\left(N_{\mathrm{c}}\right)$ from the enhanced interaction energy. At a microscopic level baryonic interactions originate from $N_{\mathrm{c}}$ permutations of color-singlet quark exchanges, and so one may well consider that this $\mathcal{O}\left(N_{\mathrm{c}}\right)$ pressure of baryonic matter is dominated by quarks even in the confined phase in which excitations on top of the Fermi surface should still be baryons. In this theoretically idealized world with $N_{\mathrm{c}} \rightarrow \infty$ the picture of quarkyonic matter is well-defined. It is important to note that not only two-body but all many-body color-singlet interactions in quarkyonic matter are of the same order $\sim \mathcal{O}\left(N_{\mathrm{c}}\right)$ according to the large- $N_{\mathrm{c}}$ counting [25].

Now, we must emphasize the importance of departing from the idealized setup and refine the deconfinement

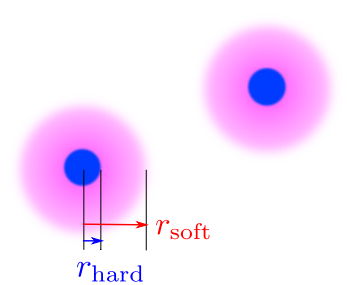

FIG. 1. Nucleons are characterized by two scales, $r_{\text {hard }}$ representing the hard core radius, and $r_{\text {soft }}$ representing the size of the surrounding meson clouds.

picture for $N_{\mathrm{c}}<\infty$. The idea of quarkyonic matter has posed an interesting question that was considered long ago in nuclear physics: the short-range repulsive hard-core interaction between nucleons can be viewed as arising from quark exchanges exactly in the same way as in the quarkyonic matter argument, while the long-range interaction via meson exchanges also involves quark-antiquark exchange mechanisms at a microscopic level. ${ }^{1}$ So, on the one hand, there appears to be no principal difference between these two types of interactions. On the other hand, as we shall point out, the separation between shortand long-distance nuclear interactions has its correspondence in a delineation of hard and soft scales in the structure of the nucleon itself, and this is the baseline for our subsequent discussion of "hard" and "soft" deconfinement.

Let us visualize our Gedankenexperiment of deconfinement. Nucleons at low energy can be viewed approximately as composed of a hard core surrounded by meson (or quarkantiquark pair) clouds as picturized in Fig. 1. Typically, the core radius is around $\sim 0.5 \mathrm{fm}$, while the radius of meson clouds is around $\sim 1 \mathrm{fm}$ as will be substantiated further in the next section. If the baryon density is so high that the hard cores begin to overlap, quark matter is unambiguously realized. We shall call this hard deconfinement of quark matter. Once hard deconfinement occurs, we can infer quantitative properties of such dense quark matter from the internal nucleon structures, as we will demonstrate for the construction of the equation of state $(\mathrm{EoS})$ in the present work.

One may also identify hard deconfinement with "valence" quark deconfinement. The baryon number distribution in a single nucleon is localized in the core region: the $N_{\mathrm{c}}$ valence quarks in the core add up to the net baryon number. In this way, especially in the large- $N_{\mathrm{c}}$ limit, hard deconfinement can be clearly defined in terms of baryon transport: in the confined phase the baryon number transport is suppressed by heavy baryon masses, while quarks can transport the baryon number once hard deconfinement occurs. Meson clouds, on the other hand, carry no net baryon number. The baryon number conductivity can be

\footnotetext{
${ }^{1}$ In this context pions are special because of their role as chiral Nambu-Goldstone bosons.
} 


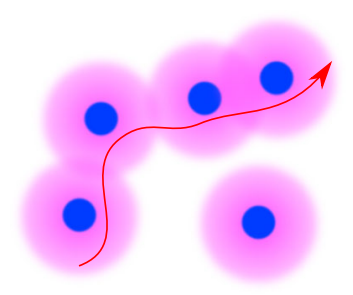

FIG. 2. If the interaction clouds are classically percolated, the quark mobility seems not restricted and quarks may classically flow over connected blocks of meson clouds.

interpreted as the heat conductivity which can therefore act as an approximate order parameter for hard deconfinement.

We point out that hard deconfinement is based on hardcore dominance. The condition for the hard-core dominance is stronger than needed for deconfinement in a more conventional sense as conjectured by the notion of quark mobility. Let us consider decreasing the baryon density and explore how the quark mobility would change. It appears that quarks (accompanied by antiquarks) can still hop from one nucleon to another through the exchange of mesons. This situation can be intuitively understood as overlaps of meson clouds as illustrated in Fig. 2. Such an interpretation of the quark mobility is, however, too naïve. The equilibrium binding of nuclear matter at the saturation density is sustained mainly by mesonic exchanges, but needless to say, nuclear matter at the saturation density is not quark matter yet. Quark exchanges inevitably occur together with antiquarks to form color-singlets, and connected blocks of meson clouds do not really signify quark liberation.

The question that we would like to address in this work is the following: there is supposedly another mechanism of quark deconfinement at lower density than hard deconfinement, which we refer to as soft deconfinement. The question is; in other words, when does a picture of individual meson exchanges between nucleons lose its meaning? If the system is in the confined hadronic phase at low density, the exchange of color-singlet mesons characterizes baryon interactions. The contraposition of this statement is that, if a meson-exchange based description is blurred, the system should be out of the confined phase. Interestingly, this argument suggests a possible relationship between soft deconfinement and quarkyonic matter. As mentioned before, quarkyonic matter has the potential energy $\sim \mathcal{O}\left(N_{\mathrm{c}}\right)$ and all $n$-body interactions are of the same order. This is exactly the situation expected in an intermediate state between nuclear and quark matter in the three-window scenario description of neutron stars [27]. Even in the real world with $N_{\mathrm{c}}=3$ we can still adopt this characterization of quarkyonic matter, namely, matter with comparable strengths of all $n$-body interactions among nucleons. From the microscopic point of view such $n$-body forces could be mediated by multimeson exchanges as sketched in Fig. 3. In this way we may well identify
FIG. 3. Schematic picture of matter with comparable strengths of all $n$-body interactions. Extended wave functions are for quarks and antiquarks. For larger $n$ a picture of individual meson exchanges would become more obscured. At short distances core interactions are mediated by quark exchanges.

quarkyonic matter in the $N_{\mathrm{c}}=3$ real world as multibody interacting matter, and we could also adopt this identification for soft deconfinement.

The regime of soft deconfinement can thus be viewed as clustering of nucleons connected by strong $n$-body interactions. Large $n$ would imply large clusters. More precisely, the clusters should be formulated in terms of wave functions of quarks and antiquarks. Mesonic clouds are to be interpreted as "sea" quarks which do not carry net baryon charge. The corresponding wave functions of quarks and antiquarks are equally distributed in space.

Such a spatial extension of wave functions is quite analogous to those of electrons in a tight-binding model. Here, based on an analogy with condensed matter physics, we are proposing a novel scenario of deconfinement. In the metallic state conduction electrons are extended in space, but a larger concentration of impurity increases the electric resistivity, and eventually the system under impurity disturbances behaves as an insulator. Then, the electron wave functions are localized in the insulating state, for which the physical mechanism is known as Anderson localization. As a matter of fact, the idea of the Anderson localization applies to the percolation problem. We emphasize that connected blocks of meson clouds in Fig. 2 are percolating classically, but this classical percolation does not necessarily lead to physical percolation of wave functions at the quantum level.

It is easily understood that the critical concentration for the onset of percolation should be larger for quantum percolation than for classical percolation. The interaction via meson exchanges opens a classical path for quarks and antiquarks to hop between nucleon sites. To build a model in the simplest way, let us consider a lattice system as schematically shown in Fig. 4. We simplify the interaction clouds into bonds connecting neighboring sites and place static nucleons (which is justified in the large- $N_{\mathrm{c}}$ limit) on sites. The bonds should be color-singlets, and this 


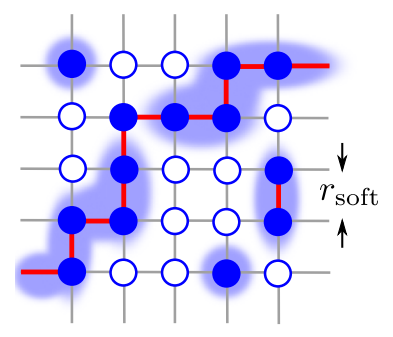

FIG. 4. Schematic picture of the classical and the quantum percolation between nucleons. Neighboring nucleons are linked by interactions shown by red bonds. The path connected by interaction bonds does not necessarily guarantee extending wave functions at the quantum level. The square lattice is only for graphical simplicity.

constraint reduces the strength of the interaction from $\mathcal{O}\left(N_{\mathrm{c}}^{2}\right)$ to $\mathcal{O}\left(N_{\mathrm{c}}\right)$. Furthermore, quarks and antiquarks are equally distributed, reflecting the nature of sea quarks associated with mesonic clouds.

We increase the baryon density by filling sites randomly, and then, soft deconfinement can be modeled as the site quantum percolation. In Fig. 4 we see a bond connected path from the left to the right edge and the classical percolation would allow quarks and antiquarks to float. In quantum physics, however, we should solve the quark and antiquark wave functions and they could be more localized than the classical path depending on the corresponding eigenenergy of the Hamiltonian. This can be easily understood from familiar examples of quantum wave functions that can have nodes. Such a prominent distinction between the classical and the quantum percolations is nicely explained in Ref. [28]. Furthermore, one essential feature of the quantum percolation is that we should consider deconfinement of sea quarks and antiquarks mode-by-mode with different eigenenergies. Therefore, our proposed quantum percolation scenario leads to a picture similar to a momentum-shell model of confinement and deconfinement as proposed in Refs. [29,30].

This paper is organized as follows. In Sec. II B we discuss the hard core and the soft surface in the nucleon structures. We introduce a chiral soliton model to demonstrate quantitative analyses. As an application we estimate the EoS of dense quark matter inferred from hard core regions of nucleon in Sec. III. Then, we shall proceed to discussions on soft deconfinement in Sec. IV. In the present paper we limit ourselves to discuss general properties of quantum percolation and make a speculative scenario of mode-by-mode soft deconfinement. Section V is devoted to conclusions.

\section{HARD AND SOFT SCALES IN THE NUCLEON}

As a preliminary exercise we shall review the internal structure of nucleon. We utilize a chiral soliton model to quantify the hard-core region where the baryon density is localized, and the soft-tail region where the meson clouds spread.

\section{A. Empirical facts and phenomenology}

At this point it is useful to give a brief summary of what is known about scales and sizes of the nucleon, in terms of the empirical radii determined by various nucleon form factors. Key quantities in this context are the proton and neutron electromagnetic form factors and their slopes at zero momentum transfer which define the mean squared charge radii. A recent new electron-proton scattering measurement [31] gives the r.m.s. proton charge radius, $\left\langle r_{p}^{2}\right\rangle^{1 / 2}=0.831 \pm 0.014 \mathrm{fm}$. A precision analysis of deuteron form factors using chiral effective field theory [32] determines the slope of the neutron electric form factor as $\left\langle r_{n}^{2}\right\rangle=-0.106 \pm 0.006 \mathrm{fm}^{2}$. The isoscalar and isovector radii of the nucleon, given by

$$
\left\langle r_{S, V}^{2}\right\rangle=\left\langle r_{p}^{2}\right\rangle \pm\left\langle r_{n}^{2}\right\rangle,
$$

have the resulting values:

$$
\sqrt{\left\langle r_{S}^{2}\right\rangle} \approx 0.77 \mathrm{fm}, \quad \sqrt{\left\langle r_{V}^{2}\right\rangle} \approx 0.89 \mathrm{fm} .
$$

The isovector charge radius reflects the interacting twopion cloud of the nucleon with its spectrum governed by the $\rho$ meson and a low-mass tail extending down to the $\pi \pi$ threshold. The isoscalar charge radius is related to the threepion spectrum [33] that is strongly dominated by the $\omega$ meson with its mass, $m_{V}=783 \mathrm{MeV}$, and its narrow width, $\Gamma=8.5 \mathrm{MeV}$. The isoscalar electromagnetic current of the nucleon is well described by the vector meson dominance phenomenology. The vector meson dominance relates the electric form factor, $G_{S}^{E}\left(q^{2}\right)$ (with $G_{S}^{E}(0)=1$ ), to a combination of $F_{B}\left(q^{2}\right)$ (i.e., the form factor of the baryon number distribution in the nucleon core) and the $\omega$ field propagation (for which the baryon density acts as a source):

$$
G_{S}^{E}\left(q^{2}\right)=\frac{F_{B}\left(q^{2}\right)}{1+\left|q^{2}\right| / m_{\omega}^{2}} .
$$

This implies,

$$
\left\langle r_{S}^{2}\right\rangle=\left\langle r_{B}^{2}\right\rangle+\frac{6}{m_{\omega}^{2}}
$$

with the mean squared radius,

$$
\left\langle r_{B}^{2}\right\rangle=-\left.6 \frac{d F_{B}\left(q^{2}\right)}{d q^{2}}\right|_{q^{2}=0}
$$

of the baryon density distribution. With the empirical input one finds, 


$$
\sqrt{\left\langle r_{B}^{2}\right\rangle} \approx 0.45 \mathrm{fm}
$$

The characteristic smallness of the radius of the baryon density distribution as compared to radius of the charge distribution underlines the proposed delineation of hard and soft scales in the nucleon: the valence quarks carrying baryon number are localized in the compact $\sim 0.5 \mathrm{fm}$ core, while quark-antiquark pairs with no net baryon number form the meson cloud at the nucleon surface.

The sizes and scales just outlined above refer to the vector currents of quarks in the nucleon. Another quantity of interest is the scalar quark density and the corresponding scalar-isoscalar form factor denoted as $\sigma\left(q^{2}\right)$. Its value at $q^{2}=0$ is identified with the pion-nucleon sigma term, $\sigma_{\pi N}$, i.e., the measure of quark mass contributions to the nucleon mass. The dispersion relation representation of $\sigma\left(q^{2}\right)$ involves the two-pion spectral function related to the $s$-wave isoscalar $\pi \pi$ scattering amplitude in combination with pion-nucleon scattering. While details of a series of investigations of $\sigma\left(q^{2}\right)$ over several decades [34-37] depend on the precise value of $\sigma_{\pi N}$, there is agreement that the radius associated with the scalar-isoscalar two-pion cloud of the nucleon is as large as $\left\langle r_{\sigma}^{2}\right\rangle^{1 / 2} \approx 1.0-1.2 \mathrm{fm}$.

The core-plus-cloud picture of the nucleon just discussed and sketched in Fig. 1 actually arises as a natural consequence of the spontaneously broken chiral symmetry of QCD at low energies [38]. The compact hard core hosting the valence quarks, and the surrounding soft surface composed of Nambu-Goldstone bosons (the multipion cloud), are the basic components of a variety of chiral models of the nucleon [39], ranging from different versions of chiral and cloudy bag models to chiral solitons. We will use the latter for orientation in order to quantify the hard and soft scales in the nucleon.

\section{B. Hard core and soft surface in a chiral soliton model}

We shall now look into the baryon structure more quantitatively. To this end we choose a chiral soliton model with $\pi, \rho$, and $\omega$ fields. For simplicity we will not quantize the soliton but rescale the results by a mass discrepancy as we will explain later. According to Refs. [40,41], we can construct baryons as the Skyrmions of $\pi$ field which is stabilized by not the Skyrme term but the coupling with $\rho$ and $\omega$ vector mesons as first considered in Ref. [42] followed shortly by Ref. [43]. We introduce the chiral fields and the vector fields as

$$
\begin{gathered}
U(\boldsymbol{r})=e^{i \tau \cdot \hat{r} F(r),} \\
\rho^{i, a}(\boldsymbol{r})=\epsilon^{i k a} \hat{\boldsymbol{r}}^{k} \frac{G(r)}{g r}, \\
\omega^{\mu}(\boldsymbol{r})=\delta^{\mu 0} \omega(r) .
\end{gathered}
$$

We determine these fields to minimize the energy, i.e., these fields should satisfy a set of equations as

$$
\begin{gathered}
F^{\prime \prime}=-\frac{2}{r} F^{\prime}+\frac{1}{r^{2}}[4(G+1) \sin F-\sin 2 F] \\
+\tilde{m}_{\pi}^{2} \sin F-\frac{3 g}{4 \pi^{2} r^{2}} \omega^{\prime} \sin ^{2} F, \\
G^{\prime \prime}=2 g^{2}(G+1-\cos F)+\frac{1}{r^{2}} G(G+1)(G+2), \\
\omega^{\prime \prime}=-\frac{2}{r} \omega^{\prime}+2 g^{2} \omega-\frac{3 g}{4 \pi^{2} r^{2}} F^{\prime} \sin ^{2} F .
\end{gathered}
$$

In what follows below we combine $r, \omega$, and $m_{\pi}$ with $f_{\pi}$ to make them dimensionless (where the dimensionless pion mass is specifically denoted by $\tilde{m}_{\pi}$ ). In the above equations only one mass scale independent of $f_{\pi}$ is the pion mass $m_{\pi}$, while the vector meson masses, $m_{\rho}^{2}=m_{\omega}^{2}=2 g^{2} f_{\pi}^{2}$, follow from the KSFR (Kawarabayashi-Suzuki [44] and Fayyazuddin-Riazuddin [45]) relation and they scale with $f_{\pi}$. From the KSFR relation we can fix $g=m_{\omega} /\left(\sqrt{2} f_{\pi}\right)=$ 6.0 (with $\sqrt{2} f_{\pi}=130.41 \mathrm{MeV}$ and $m_{\omega}=783 \mathrm{MeV}$ ). We solve these differential equations under the boundary conditions:

$$
F(0)=\pi, \quad F(\infty)=0,
$$

which is required to quantize the baryon number $B=1$. The vector mesons should satisfy the following boundary conditions:

$$
\begin{aligned}
G(0) & =-2, \quad G(\infty)=0, \\
\omega^{\prime}(0) & =0, \quad \omega(\infty)=0 .
\end{aligned}
$$

We note that $F(0), G(0)$, and $\omega^{\prime}(0)$ are initial conditions and we adjust the rest of initial conditions, $F^{\prime}(0), G^{\prime}(0)$, and $\omega(0)$, to realize the asymptotic behavior, $F(\infty), G(\infty)$, $\omega(\infty) \rightarrow 0$. We show the numerical solutions for $F(r)$, $-G(r)>0$, and (dimensionlessly scaled) $\omega / f_{\pi}$ in Fig. 5 .

With these field profiles we can immediately compute physical quantities. In this way we can concretely demonstrate the baryon structure and exemplify a hard core surrounded by a soft tail. The baryon number density $\rho_{\mathrm{B}}(r)$ should be localized in the hard core region and we can see this from an explicit expression:

$$
\rho_{\mathrm{B}}(r)=-\frac{1}{2 \pi^{2} r^{2}} F^{\prime}(r) \sin ^{2} F(r),
$$

which leads to the properly quantized baryon number; $B=4 \pi \int d r r^{2} \rho_{\mathrm{B}}(r)=1$. The pion clouds can be characterized by the isoscalar charge density given by 


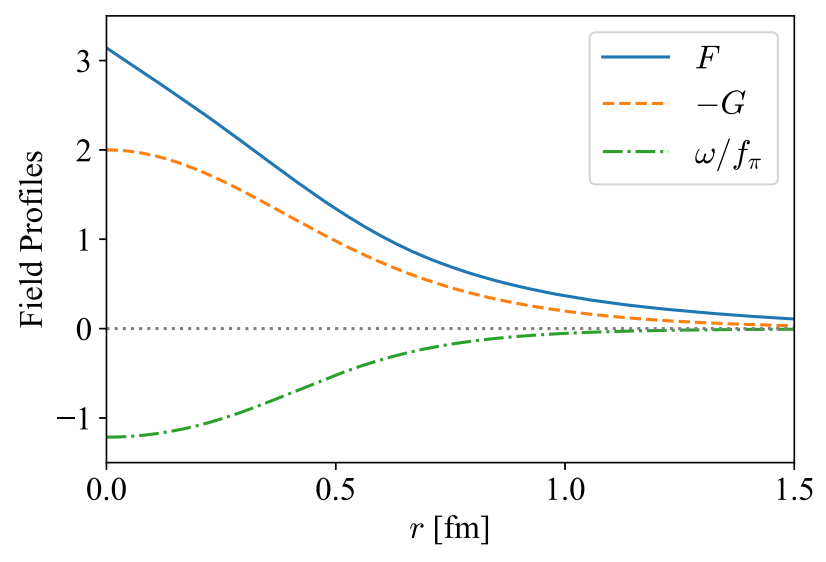

FIG. 5. Numerical solutions of $F(r)$ (solid curve), $G(r)$ (dashed curve), and $\omega(r)$ (dot-dashed curve) scaled dimensionlessly with $f_{\pi}$.

$$
\rho_{\mathrm{S}}(r)=-2 g f_{\pi}^{2} \omega
$$

We show the numerical behavior of $4 \pi r^{2} \rho_{\mathrm{B}}(r)$ and $4 \pi r^{2} \rho_{\mathrm{S}}(r)$ in Fig. 6, where $\rho_{\mathrm{B}}(r)$ gives the information on the core extension and $\rho_{\mathrm{S}}(r)$ has a longer tail which behaves asymptotically as $\sim e^{-3 m_{\pi} r}$. Using these distributions we can estimate the r.m.s. radii as

$$
\begin{aligned}
& \sqrt{\left\langle r_{B}^{2}\right\rangle}=\left(\frac{\int_{0}^{\infty} d r r^{4} \rho_{\mathrm{B}}(r)}{\int_{0}^{\infty} d r r^{2} \rho_{\mathrm{B}}(r)}\right)^{1 / 2} \approx 0.49 \mathrm{fm}, \\
& \sqrt{\left\langle r_{S}^{2}\right\rangle}=\left(\frac{\int_{0}^{\infty} d r r^{4} \rho_{\mathrm{S}}(r)}{\int_{0}^{\infty} d r r^{2} \rho_{\mathrm{S}}(r)}\right)^{1 / 2} \approx 1.03 \mathrm{fm} .
\end{aligned}
$$

These numbers are close to those of Eqs. (2) and (6). It is conceivable to relate $\sqrt{\left\langle r_{B}^{2}\right\rangle}$ to $r_{\text {hard }}$ and $\sqrt{\left\langle r_{S}^{2}\right\rangle}$ to $r_{\text {soft }}$.

It should be noted here that $\rho_{\mathrm{B}}(r)$ represents the net baryon distribution and thus the hard core should be dominated by "valence" quark degrees of freedom, as already mentioned. In contrast, the interaction clouds carry no net baryon charge, and the interaction tail should be dominated by "sea" quark degrees of freedom. This difference is a key ingredient to distinguish two states of deconfinement in later discussions.

\section{HARD DECONFINEMENT AND THE EQUATION OF STATE}

Here we discuss the nature of hard deconfinement and its implication to the EoS of dense quark matter. As we already mentioned, the quark mobility itself could be enhanced even before the hard cores touch each other, and strictly speaking, there is no transition of deconfinement associated with hard deconfinement. Still, it is convenient to think of matter in the regime of hard deconfinement.

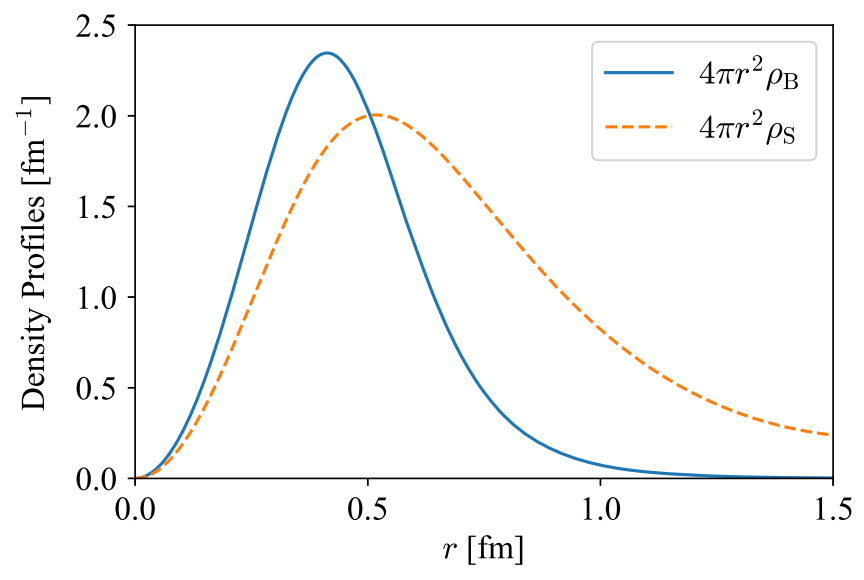

FIG. 6. Baryon and isoscalar charge density distributions as functions of $r$ multiplied by $4 \pi r^{2}$.

The term hard deconfinement is used to consider a state of matter dominated by properties of the baryon hard cores. Let us first give a rough estimate of the relevant density for hard deconfinement. We shall assume the closest packed state, i.e., either the hexagonal close-packed (hcp) or the face-centered cubic (fcc) lattice, in which the filling rate is $74 \%$. As we read from Fig. 6 the hard core radius is around $r_{\text {hard }} \sim 0.5 \mathrm{fm}$. When the closest packed state occurs, the baryon density corresponding to the filling fraction is

$$
\sim 0.74 \times\left(\frac{4}{3} \pi r_{\text {hard }}^{3}\right)^{-1} \simeq 1.4 \mathrm{fm}^{-3}
$$

which is $\sim 8.3 \rho_{0}$ in the unit of the normal nuclear density; $\rho_{0} \sim 0.17 \mathrm{fm}^{-3}$. We note that this density, $\sim 8.3 \rho_{0}$, is estimated for the closest packed state, and so this should be taken as the limiting value below which hard deconfinement should be realized. It is a sufficient condition that the hard cores (as rigid spheres) touch each other in the static closest packed state. In reality nucleons have Fermi motion, and their hard cores are to be replaced by continuous distributions as seen in Fig. 6. The critical density for hard deconfinement as a continuous transition can thus be lower than Eq. (19) by some factor.

Hard deconfinement provides us with an interesting opportunity to make a quantitative assessment of bulk matter from a single baryon. It is conceivable for thermodynamic pressure of bulk matter to be approximated by mechanical pressure in a hard core, $p(x)=\left\langle N\left|T_{i i}(x)\right| N\right\rangle$, where $|N\rangle$ is a nucleon state and $T_{\mu \nu}$ is the energy momentum tensor. To separate the surface effects specific to an isolated nucleon, we focus on the pressure near the center of the hard core. Within the framework of the model explained in Sec. II B, we can compute the pressure and the energy density. Their expressions (made dimensionless divided by $f_{\pi}^{4}$ ) are found to be 


$$
\begin{gathered}
p(r)=-\frac{1}{6} F^{\prime 2}-\frac{\sin ^{2} F}{3 r^{2}}-\frac{2}{3} \frac{(G+1-\cos F)^{2}}{r^{2}}+g^{2} \omega^{2}+\frac{1}{6} \omega^{\prime 2}+\frac{G^{2}}{3 g^{2} r^{2}}+\frac{G^{2}(G+2)^{2}}{6 g^{2} r^{4}}-\tilde{m}_{\pi}^{2}(1-\cos F), \\
\epsilon(r)=\frac{1}{2} F^{\prime 2}+\frac{\sin ^{2} F}{r^{2}}+2 \frac{(G+1-\cos F)^{2}}{r^{2}}-g^{2} \omega^{2}-\frac{1}{2} \omega^{\prime 2}+\frac{G^{\prime 2}}{g^{2} r^{2}}+\frac{G^{2}(G+2)^{2}}{2 g^{2} r^{4}}+\frac{3 g}{4 \pi^{2} r^{2}} \omega F^{\prime} \sin ^{2} F+\tilde{m}_{\pi}^{2}(1-\cos F) .
\end{gathered}
$$

We can check from the Virial theorem that the above pressure satisfies; $\int d r r^{3} p(r)=0$ as it should. We performed numerical calculations and present $p(r)$ and $\epsilon(r)$ (multiplied by $4 \pi r^{2}$ ) in Fig. 7 where we rescaled the energy density by a factor 0.1 to make it comparable to the pressure. The characteristic feature of the pressure distribution inside the nucleon is its combination of a positive core pressure and a negative pressure at the surface [46,47], adding up to overall zero pressure to maintain equilibrium in the nucleon ground state. Such a pressure profile is verified, at least qualitatively, in deeply virtual Compton scattering measurements [48].

From these results one can infer the relation between the mechanical pressure and energy density in the core region of the nucleon, which may serve as a reasonable approximation for the EoS of quark matter near the closest packed density (19). One might care about differences between symmetric nuclear matter and neutron matter, but in such an extremely high-density regime of our interest the physical properties are to be dominated by the strong interaction and the $\beta$-equilibrium condition would be not essential.

In the present framework we must be careful of the mass scale in executing this program for the EoS construction. As discussed in the previous work [40,41], this chiral soliton model overestimates the baryon mass which is given by the integration of the energy density. It is known that this mass discrepancy would be reduced if the soliton is

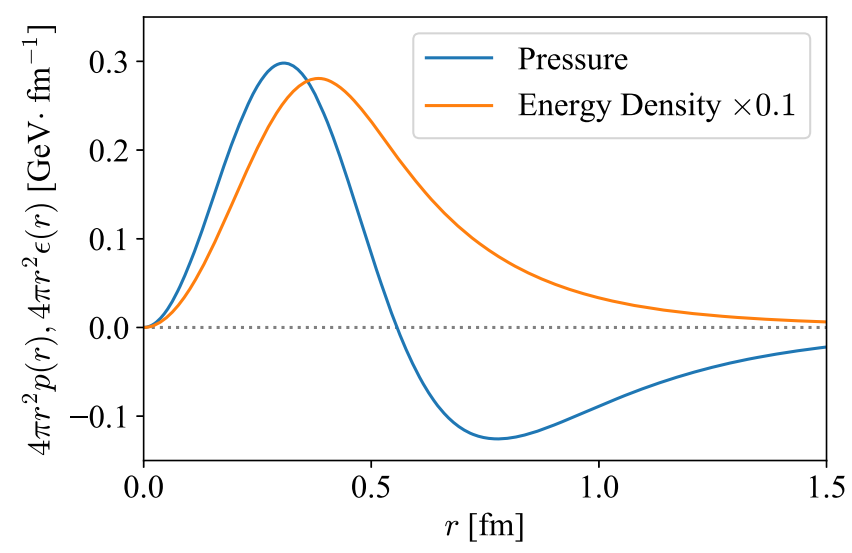

FIG. 7. Pressure and energy density distributions as functions of $r$ multiplied by $4 \pi r^{2}$. To make the comparison easier, the energy density is rescaled by a factor 0.1 . quantized (i.e., rotated with spin and isospin). Here, our main purpose is not to study the chiral soliton model itself but to demonstrate the idea, so we shall adopt a quick prescription: we rescale the results simply by the ratio, $\chi$, between the physical nucleon mass and the model output. That is, we introduce a ratio parameter as

$$
\chi=\frac{(\text { physical mass })}{(\text { model mass })} \approx \frac{940 \mathrm{MeV}}{1460 \mathrm{MeV}} \approx 0.64 .
$$

Then, we should make the following rescaling:

$$
\epsilon(r) \rightarrow \chi \epsilon(r), \quad p(r) \rightarrow \chi^{-1} p(r) .
$$

The above is the consistent rescaling in such a way not to modify the form factors. In other words, given the nucleon form factors $A\left(q^{2}\right)$ and $D\left(q^{2}\right)$ associated with the components of the energy-momentum tensor, the energy density is proportional to the mass, while the pressure is inversely proportional to the mass (see a review [49] for explicit expressions). One might have thought that the model parameters can be readjusted to fit the baryon mass, but this would significantly affect the charge radius. If the form factors stay intact leaving the charge radius unchanged, the rescaling procedure should yield physically more sensible results than readjusting the model parameters.

Figure 8 presents our results for the equation of state, $p(\epsilon)$, of dense quark matter in the hard core region of the nucleon, compared to several proposed EoSs that are consistent with empirical properties of neutron star matter. We label our results, the rescaled $p(r)$ and $\epsilon(r)$, as "Nucleon EoS" and mark different radial coordinate scales in the nucleon core, $r=0.2 \mathrm{fm}$ to $0.5 \mathrm{fm}$, with crosses in Fig. 8. The fast-dropping behavior at $r \gtrsim 0.5 \mathrm{fm}$ reflects the negative pressure at the nucleon surface, physically interpreted as resulting from confining forces and the inwardbound pressure of the meson cloud.

For the neutron star based equations-of-state in Fig. 8, $\chi$ EFT refers to the EoS from the chiral effective theory [50] and QHC18 from Ref. [27], and SLy4 from Ref. [51]. DL shows the EoS deduced from the observation data analyses using the deep learning [52]. The EoS data labelled by $\chi$ FRG is taken from Refs. [53,54]. We note that the EoS bound from the deeply virtual Compton scattering on the proton was previously discussed in a similar way in 


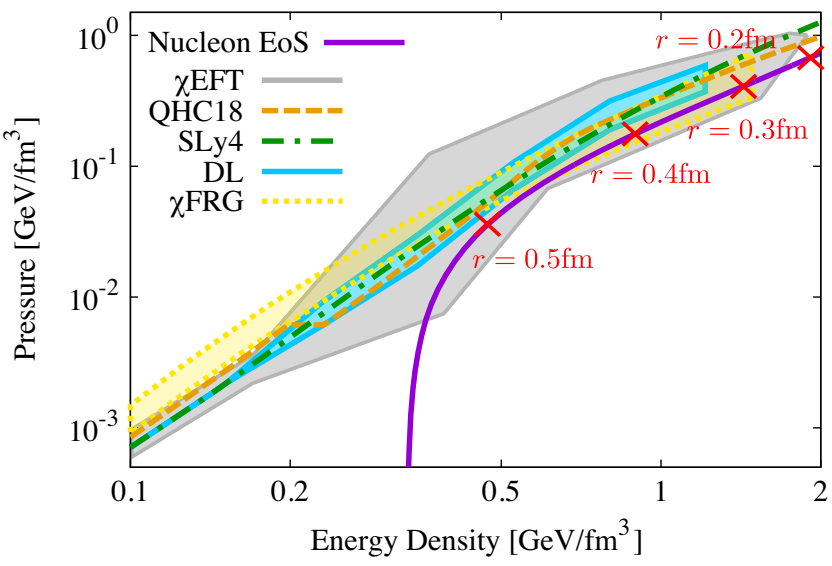

FIG. 8. EoS of dense quark matter from the hard deconfinement scenario (Nucleon EoS) and empirical EoSs from other approaches.

Ref. [55]. For $r<0.5 \mathrm{fm}$, remarkable agreement is seen between our (free) nucleon EoS and the sets of dense neutron star matter equations-of-state. Assuming that the onset of hard deconfinement appears at $r$-scales in the range $r=0.5-0.4 \mathrm{fm}$ (corresponding to baryon densities $\sim 4-7 \rho_{0}$ according to Fig. 6), this implies that hard deconfinement can occur at significantly lower density than the limiting estimate (19).

Before closing this section we mention the possibility of partial chiral symmetry restoration at high density. So far we have been looking at single baryon properties in vacuum, but interactions with surrounding baryons are expected to change its properties in a high-density environment. The simplest way to implement this effect is to reduce the chiral order parameter, $f_{\pi}$, in the chiral soliton model. Although the model has two independent mass scales, $f_{\pi}$ and $m_{\pi}$, we numerically found that the solutions of the chiral soliton model scale with $f_{\pi}$ in a good approximation. This means that, if $f_{\pi}$ decreases to $f_{\pi}^{*}$ in a medium, $\epsilon(r)$ and $p(r)$ change as

$$
\epsilon(r) \rightarrow\left(\frac{f_{\pi}^{*}}{f_{\pi}}\right)^{4} \epsilon(r), \quad p(r) \rightarrow\left(\frac{f_{\pi}^{*}}{f_{\pi}}\right)^{2} p(r) .
$$

In Fig. 8 these modifications shift $\epsilon(r)$ by a factor along the horizontal axis, while the vertical axis is given by the logarithmic scale and the vertical shift of the nucleon EoS curve is not by a factor but nearly by an offset. Therefore, the EoS would be stiffer if in-medium $f_{\pi}^{*}$ gets smaller with increasing density. This stiffening, with $f_{\pi}^{*} \sim 0.8 f_{\pi}$ for example, improves the agreement between "nucleon EoS" and the others in the high density region in Fig. 8. We note that the ratio $\chi$ might in principle also be density dependent, but this dependence should be approximately negligible. This is because both the physical mass and the model mass should be affected by partial chiral symmetry restoration and their ratio is expected to be unchanged.

\section{SOFT DECONFINEMENT AS QUANTUM PERCOLATION}

In this section we discuss the scenario of soft deconfinement. We begin with a brief description of classical percolation and then proceed to a concrete model of quantum percolation. We summarize the basic properties of quantum percolation and translate them in the context of quark liberation in dense baryonic matter. Most important is the observation of energy dependent percolation which leads to a novel picture of mode-by-mode delocalization of quark wave functions, akin to a recently proposed momentum-shell model in the quarkyonic matter picture.

\section{A. Classical percolation}

As we did previously for hard deconfinement, we shall begin with an order estimate for the critical concentration in percolating baryonic matter. The idea to interpret quark deconfinement as percolating baryons is traced back to pioneering works, see Ref. [56] for example, in which the "hadron solid" could be considered in terms of weakly coupled quark matter. This is a prototype of the percolation model of quark deconfinement. A more refined picture was discussed in Ref. [57], in which the percolating density was estimated as $\sim 5.5 \rho_{0}$. The $N_{\mathrm{c}}$ dependence of percolation for tightly packed baryons was discussed in Ref. [58]. In our language these percolation models with hard cores rather correspond to hard deconfinement.

The characteristic scale in the soft deconfinement scenario is given by a typical length scale of the mesonic clouds rather than the hard cores. Such a picture was also considered, for example, in Ref. [59]. The essential argument in Ref. [59] is that the relative importance of multibody interactions is given parametrically by $n /\left(2 m_{\pi}\right)^{3}$ where $n$ represents the baryon density, and this approaches $\sim \mathcal{O}(1)$ when $n$ gets larger, which is also emphasized in the recent review [27]. We note, however, that the relevant length scale is not necessarily $\sim 1 /\left(2 m_{\pi}\right)$ in reality, and the multibody interactions in the $\chi$ EFT suggest that the relevant scale should be 1.1-1.3 fm [60], which is consistent with the r.m.s. radius of the scalar-isoscalar nucleon form factor [35] (depending on the value of the pion-nucleon sigma term). Also, if the relevant scale is related to the Compton wavelength corresponding to a spectral maximum in the scalar-isoscalar channel, it would be $1 / m_{\sigma} \sim 0.4 \mathrm{fm}$. The soft scale has the largest uncertainty in this picture and in the present consideration we shall choose $r_{\text {soft }} \sim 0.7 \mathrm{fm}$ for the moment, close to the value in Eq. (2).

Now, let us concretize the percolation picture by the following modeling. We assume the Born-Oppenheimer approximation; (i) Baryons move at velocity $p_{F} / m_{B} \sim$ $\mathcal{O}\left(N_{\mathrm{c}}^{-1}\right)$, much slower than quarks at velocity of $\mathcal{O}(1)$. (ii) Quark wave functions solved for a given quasistatic configuration of baryons. (iii) Physical quantities estimated as a result of averaging over the slow baryon dynamics. For simplicity we replace the time averaging procedure in 
(iii) by the ensemble average. Within this framework we shall give a quick estimate of the critical percolating density adopting a three-dimensional model of sphere percolation. In this model there are sites connected by networks (i.e., connected bonds), and each site is either occupied by a particle (baryon) with the probability $p$, or empty with the probability $1-p$. We note that the model allows one baryon per site and this feature is consistent with the hard core repulsion. The probability $p$ can be easily translated into the particle density.

The classical percolation in this three-dimensional model is characterized by the existence of connecting networks between different boundaries of the whole system. The critical filling fraction $p_{\mathrm{c}}$ is defined by a condition that a cluster connecting two boundaries (e.g., $x=-\infty$ and $x=+\infty$ ) begins to be formed. It is known from Ref. [61] that the critical filling fraction is $p_{\mathrm{c}} \sim 0.34$ in this model (other types of lattice models take a similar value of the critical filling fraction). Thus, the critical density for classical percolation would be

$$
\sim 0.34 \times\left(\frac{4}{3} \pi r_{\text {soft }}^{3}\right)^{-1} \simeq 0.24 \mathrm{fm}^{-3}
$$

assuming $r_{\text {soft }} \sim 0.7 \mathrm{fm}$. We emphasize that this estimate is just for qualitative considerations: if $r_{\text {soft }}$ were slightly changed by hand, the above number would quantitatively differ. With this caution in mind, using Eq. (25) the critical density for percolation would be $\sim 1.4 \rho_{0}$. It is obviously unlikely to expect quark matter to appear at such low density.

We note that the meson clouds saturate the system at $p=1$ which corresponds to $\rho \sim 4.2 \rho_{0}$. At this density baryons may be still nonrelativistic, provided that meson clouds do not strongly limit the motion of hard cores. ${ }^{2}$ Toward hard deconfinement at $\rho \sim 8.3 \rho_{0}$, hard core repulsions exclude volumes available for a baryon and lifts up the momenta, making the baryon relativistic. This is beyond the Born-Oppenheimer descriptions. The excluded volume thermodynamics for baryons was discussed in Ref. [30].

\section{B. Quantum percolation}

Quantum mechanically, the availability of classical quark paths would not guarantee percolated wave functions, i.e., wave functions delocalized in one arbitrary direction connecting two boundaries. This is because there may be destructive interferences and nodes appearing in quantum amplitudes. To consider such quantum effects, let us briefly review a simple quantum percolation model

\footnotetext{
${ }^{2}$ For the combined effects of scalar-isoscalar $(\sigma)$ boson and $\omega$ meson clouds, each contribution is $\mathcal{O}\left(N_{\mathrm{c}}\right)$, but they largely cancel. Hence we assume that short-range repulsion sets in at the hard scale $\sim r_{\text {hard }}$.
}

(see Ref. [62] for a comprehensive book; we refer to results in Ref. [63]). A typical toy model is defined by the following Hamiltonian of the tight binding model:

$$
H=\sum_{n}|n\rangle \varepsilon_{n}\left\langle n\left|+\sum_{n \neq m}\right| n\right\rangle V_{n m}\langle m|,
$$

where $|n\rangle$ denotes a state with a quark occupying site $n$. The term involving $V_{n m}$ describes quark hopping between sites $n$ and $m$. For the site percolation problem the simplest choice would be $V_{n m}=-V(V>0)$ for the nearest neighbor sites and $V_{n m}=0$ otherwise. The background baryon distribution is treated classically and specified by $\varepsilon_{n}$. Each site $n$ is occupied by a baryon with the probability $p$ or left empty with the probability $1-p$. The site energies of quarks, $\varepsilon_{n}$, depend on whether the site $n$ is occupied by the baryon or not. Let us introduce a notation $\varepsilon_{\text {on }}$ to represent the site energy of quarks at occupied site, that is, a quark energy as a part of placed baryon. If the site is left empty, the site energy is $\varepsilon_{\text {off }}$. In this way, $\varepsilon_{n}$ is generated by the following probability distribution:

$$
P\left(\varepsilon_{n}\right)=p \delta\left(\varepsilon_{n}-\varepsilon_{\mathrm{on}}\right)+(1-p) \delta\left(\varepsilon_{n}-\varepsilon_{\mathrm{off}}\right) .
$$

We set $\varepsilon_{\text {off }} \rightarrow \infty$ with which quarks cannot penetrate into empty sites. We note that this limit is common in quantum percolation problems (for large but finite $\varepsilon_{\text {off }}$, see, e.g., Refs. $[28,63])$. Under this limit the classical percolation should be a necessary condition for the quantum percolation; the critical concentration for quantum percolation, $p_{\mathrm{c}}$, must satisfy $p_{\mathrm{q}} \geq p_{\mathrm{c}}$. The eigenenergy $E$ of the Hamiltonian for this single particle problem is to be regarded as the kinetic energy of a (nonrelativistic) quark. The kinetic energy thus depends on the baryon cluster size. Let us consider two extreme examples. For a quark localized in a single baryon the eigenenergy is $\varepsilon_{\mathrm{on}}$ and from the uncertainty relation this is the high energetic case. Conversely, for completely percolated baryons with $p=1$, the eigenstates are plane-waves and the eigenenergies are $E(k)=\varepsilon_{\mathrm{on}}-2 V \sum_{i=x, y, z} \cos \left(k_{i}\right)$. Here, let us choose the energy offset so that $\varepsilon_{\text {on }}=6 \mathrm{~V}$ and $E(k)=4 V \sum_{i=x, y, z} \sin ^{2}\left(k_{i} / 2\right)$. This choice is physically reasonable for our purpose and the kinetic energy vanishes as $k_{i} \rightarrow 0$, while $\varepsilon_{\text {on }}=0$ is a usual choice in condensed matter literatures on quantum percolation.

Since the quantum interference is sensitive to the wavelength, $p_{\mathrm{q}}$ is a function of $E / V$ and further depends on geometrical site structures (i.e., square lattice, triangular lattice, continuum spheres, etc). In Fig. 9, we illustrate simple examples to exemplify a "localized" wave function. For a clear demonstration purpose let us consider onedimensionally aligned baryons from one to three. The numbers attached to each site are the amplitudes of the quark wave functions. When the amplitudes of two neighboring domains have opposite signs, there must be a node between them. For example, with the two baryon 


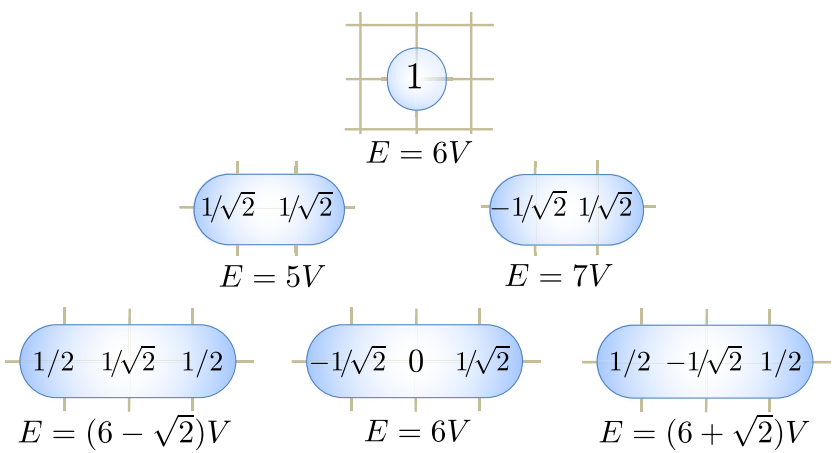

FIG. 9. Examples of single particle states for one-dimensionally aligned baryon configurations. The numbers attached to sites are the quantum amplitudes. The quark eigenenergy on an isolated baryon is $\varepsilon_{\text {on }}=6 \mathrm{~V}$. With two-baryon connected cluster (as shown in the middle row) a more extended wave function is allowed and the lowest quark eigenenergy is lowered. The threebaryon cluster (as shown in the bottom) has a state of eigenenergy $6 V$ for which a node separates the cluster into two "localized" wave functions.

background (as shown in the middle of Fig. 9) the quark wave functions have zero and one node, respectively, with the eigenenergies $5 \mathrm{~V}$ and $7 \mathrm{~V}$. Then, we would call such a state with $E=7 \mathrm{~V}$, which is partitioned into two, the "localized" state. More precisely speaking, we define localized states as a finite-amplitude domain surrounded by vanishing amplitudes (or exponentially suppressed amplitudes for more general continuum models). If the whole system is just three sites and all three sites are occupied by baryons, the quark state as shown in the left bottom in Fig. 9 is delocalized over the whole system and this is our definition of soft deconfinement. In the bottom of Fig. 9 the central figure shows a "localized" state with one node. Interestingly, this state has $E=\varepsilon_{\text {on }}=6 \mathrm{~V}$, the same energy as the isolated single baryon case (as shown in the top of Fig. 9). The far-right figure is the most energetic state with two nodes. Again, we emphasize that the quantum

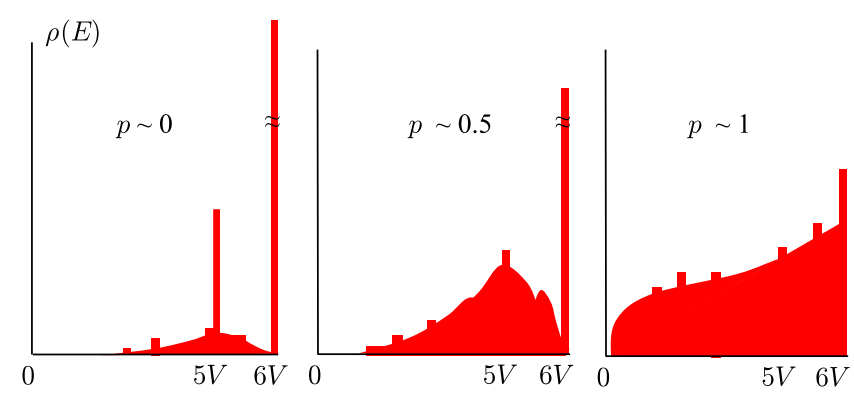

FIG. 10. Schematic histograms of $\rho(E)$ for various baryon configurations as a function of eigenenergy $E$. For dilute systems with $p \sim 0$, isolated single baryons are dominant and states with $E=\varepsilon_{\text {on }}=6 \mathrm{~V}$ are found the most frequently. For larger $p$ the baryon cluster size grows up and states at smaller $E$ with larger spatial extension develop. interference of reflected waves with boundaries is essential to create the zeros in the wave functions.

Shown in Fig. 10 are schematic histograms of quark eigenstates for various baryon configurations, namely, the density of states $\rho(E)$ as a function of the eigenenergy $E$. We note that a sum rule, $\int d E \rho(E)=1$, should hold for a single particle state. In the dilute regime at $p \sim 0$ most baryons are isolated, and eigenstates with the energy $E \simeq$ $\varepsilon_{\mathrm{on}}=6 \mathrm{~V}$ are dominant. The first nontrivial baryon configuration is a two-baryon cluster for which the eigenenergy is $\varepsilon_{\mathrm{on}} \pm V=6 V \pm V$. Hence, the histogram is expected to have peaks around $5 \mathrm{~V}$ and $7 \mathrm{~V}$ (in Fig. 10 the $E>6 V$ region is not shown as it should be symmetric from the reference at $E=6 \mathrm{~V}$ ). As $p$ increases (i.e., the baryon density increases), configurations with isolated baryons would be less populated, but subclusters of wave function are formed within the classical baryon clusters. Some of subclusters make contributions to $E=6 \mathrm{~V}$ and the peak at $E=6 V$ should persist up to $p \sim 1$.

As long as subclusters appear, quarks are still localized, even though they could flow from one baryon to another. Therefore, soft seconfinement is defined as complete delocalization of the quark wave function. For $\varepsilon_{\text {off }} \rightarrow \infty$ as is the case here, it has been conjectured that localized states should appear at $E=6 \mathrm{~V}$ until the concentration reaches $p \rightarrow 1$. In contrast, for $\varepsilon_{\text {off }}<\infty$ that allows for quantum tunneling, the $E=6 \mathrm{~V}$ state could be completely delocalized at $p<1$.

When the baryon density or $p$ gets larger, the typical spatial extension of baryon clusters and thus quark wave functions would be larger. This means that the lowest eigenenergy can be lowered and softer quark components would be involved at larger baryon density. Eventually, at the critical value of density or $p$, the wave function is delocalized in an arbitrary direction. The eigenenergy reaches $E=0$ when quarks are delocalized in all directions, and this is possible only for $p=1$, as states with $p<1$ are accompanied by vacancy of baryon clusters and it would lift up $k_{i}$ from zero and thus $E>0$ inevitably.

Figure 11 shows a schematic phase diagram of percolation (for recent numerical studies, for example, see Fig. 3 in Ref. [64]); the critical concentration of quantum percolation, $p_{\mathrm{q}}(E)$, as a function of the eigenenergy $E$ and its classical counterpart, $p_{\mathrm{c}}$, which is independent of $E$ but solely determined by the geometrical site-bond networks. For a given density or $p$, modes with $p<p_{\mathrm{q}}(E)$ are localized (they may be extended over a wider range than a single baryon, but not delocalized over the whole system), while modes with $p>p_{\mathrm{q}}(E)$ can be delocalized and deconfined. As we mentioned before, the modes at $E=$ 0 and $6 V$ are somewhat special and can get delocalized only at $p \rightarrow 1$. The behavior of $p_{\mathrm{q}}(E)$ in the small $E$ region is called the mobility edge trajectory and a relation between $p_{\mathrm{q}}(E)$ and $\rho(E)$ are known [65]. The minimum plateau of $p_{\mathrm{q}}(E)$ away from $E=0,6 V$ reads $p_{q}(E) \gtrsim 1.3 p_{\mathrm{c}}$ 


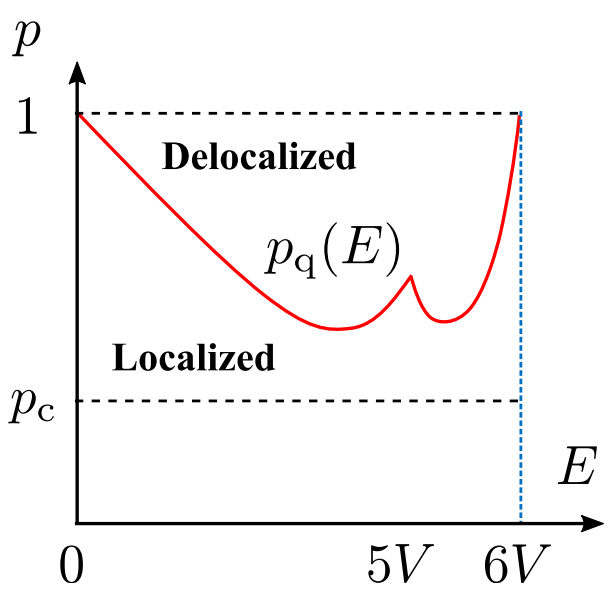

FIG. 11. Schematic phase diagram of quantum percolation; the quantum critical concentration $p_{\mathrm{q}}$ as a function of the eigenenergy $E$ and the classical critical concentration $p_{\mathrm{c}}\left(<p_{\mathrm{q}}\right)$ that is independent of $E$. The minimum is around $E / V \sim \mathcal{O}(1)$. Cusps may appear corresponding to the molecular states. The $E=0$ state would be realized only when baryons occupy all the sites, and thus $p_{\mathrm{q}}(E \rightarrow 0) \rightarrow 1$. The $E=6 V$ states are localized for any $p<1$ since single particle states get localized as long as finite cluster boundaries remain in the system.

typically. Therefore, if we adopt Eq. (25) for the critical density $\sim 1.4 \rho_{0}$ in the classical percolation picture, the quantum effects would raise it up to $\gtrsim 1.8 \rho_{0}$.

Here, let us summarize our considerations based on the quantum percolation model in the context of nuclear and quark matter. Actually, the quantum percolation model provides us with useful insights as follows.

First, the histograms of $\rho(E)$ as in Fig. 10 quantify how the quark eigenstates change as baryon clusters merge at various baryon densities. This way of understanding matter implicitly assumes duality between baryons and quarks. The point is that $\rho(E)$ carries information on quarks for such many-body systems of baryons. As the density increases, the average kinetic energy of baryons should increase, and at the same time, a larger baryon cluster would allow for quarks with smaller average kinetic energies. For soft deconfinement quark momentum eigenstates would form natural bases to characterize the nature of localization/delocalization of quarks. It is likely that the changes in $\rho(E)$ occur continuously with increasing density, so that the quantum percolation takes place mode-bymode gradually. This is a microscopic description of the quark-hadron continuity (apart from symmetries and gap energies). Because $\rho(E)$ is positive definite, the gauge average would not wash $\rho(E)$ out, and a gauge-invariant characterization would be in principle possible.

Second, localization over baryon clusters can be driven by quantum interference effects. This observation happens to be consistent with a conventional view of quark confinement. In QCD it has been established that the strong coupling limit of the theory should confine quarks due to randomness of gluons. In the present study we saw that simple configurations as in Fig. 9 exhibit rich contents in physics at the quantum level. There are various sources for randomness on top of baryon configurations, and it would deserve more investigations whether gluon fluctuations strengthen/weaken our proposed scenario. This question is beyond our current scope, and we just mention possibly related preceding works, Refs. [66-68], in which chiral symmetry breaking and Anderson localization have been discussed.

\section{Clustering and delocalization}

Finally we mention a possibility of the quantum percolation picture to give us a clue to understand quarkyonic matter [26] better from the nuclear point of view. Quarkyonic matter is well defined only in the limit of $N_{\mathrm{c}} \rightarrow \infty$ in which gluons are unscreened due to $1 / N_{\mathrm{c}}$ suppression of quark loops and quarks are still confined. Then, one may encounter a conceptual question; the baryon Fermi sea or the quark Fermi sea, which of them should be a more suitable starting point for quantitative estimates. The presence of confining forces via unscreened gluons would suggest the baryonic description, but the pressure turns out to be $\mathcal{O}\left(N_{\mathrm{c}}\right)$, and one should account for quark degrees of freedom while keeping track of the identity of baryons. For this reason with two seemingly conflicting aspects, it has been argued that the Fermi sea should be composed of quarks, and any excitations on top of it should be confined.

It is indeed possible to form a color-singlet Fermi sea by filling all colored states with quarks. The quarkyonic matter scenario presumes $N_{\mathrm{c}}$-particle correlations near the Fermi surface which form a color-singlet, i.e., a baryonic composite. Altogether, this picture leads to a model of the momentum space shell $[29,30]$. However, it seems counterintuitive to postulate quarks at lower momenta and baryons at larger momenta.

Now, let us discuss such a possible momentum shell in our language of the mode-by-mode deconfinement. The schematic illustration is shown in Fig. 12 where the occupation function, $f_{\text {quark }}(k)$, for quarks with momenta $k$ is also sketched. In the dilute regime with $\rho \ll r_{\text {soft }}^{-3}$, the isolated baryons are dominant as shown in the far left panel of Fig. 12. The quark momentum distribution is characterized merely by quark compositions inside each baryon. Therefore, $f_{\text {quark }}(k)$ should have a support up to $k \sim r_{\text {soft }}^{-1} \sim \Lambda_{\mathrm{QCD}}$. With increasing baryon density, as long as baryons are isolated, $f_{\text {quark }}(k)$ is simply piled up without changing its shape itself.

As the baryon density increases further, baryons can be clustered, as confirmed, for example, in numerical simulations of quantum molecular dynamics [69]. This happens at the density, $\rho \sim r_{\text {soft }}^{-3}$, parametrically. Nucleons are still distributed dilutely at the normal nuclear density, but eventually, the classical and the quantum percolation of 


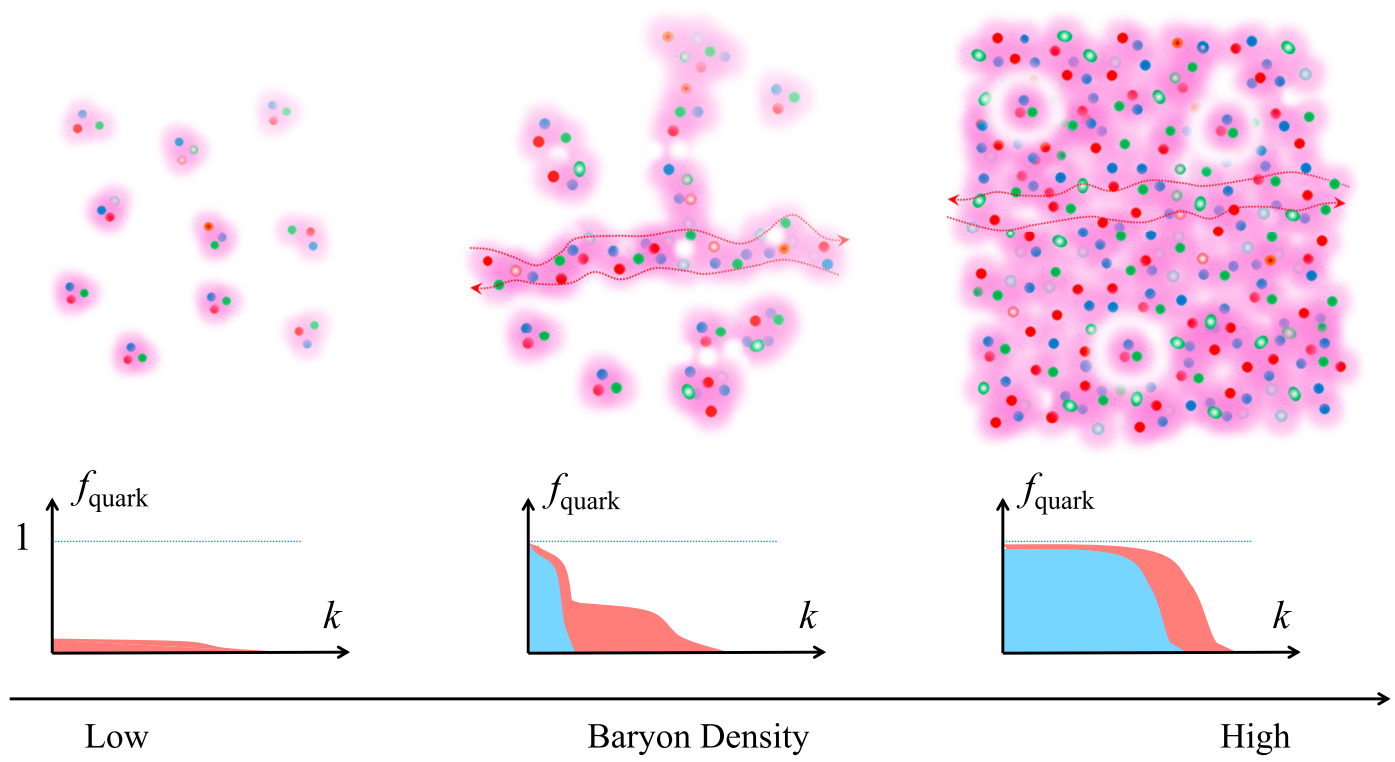

FIG. 12. Graphical representation of Soft and hard deconfinement based on the percolation picture. The occupation function, $f_{\text {quark }}(k)$, for quarks with momenta $k$ is also schematically illustrated. The red (blue) area in $f_{\text {quark }}(k)$ indicates the contributions from localized (delocalized) modes.

baryon clusters would be realized beyond a certain critical density of soft deconfinement, as illustrated in the middle panel of Fig. 12. On such percolated clusters of baryons, the quark wave functions can be delocalized with low momentum components. Then, these delocalized states would substantially contribute to $f_{\text {quark }}(k)$ in small $k$ regions as indicated by blue area in the middle panel of Fig. 12. Meanwhile, localized states associated with isolated baryons and small-sized baryon clusters still make nonzero contributions to $f_{\text {quark }}(k)$, but percolating quarks emerge from the small- $k$ regions as the cluster domains get larger. It should be noted that we do not necessarily assume inhomogeneous baryonic states. We should recall that our picture is based on the Born-Oppenheimer approximation, and the true ground state properties are obtained after taking the average over baryon configurations. The uniformity is recovered through the averaging procedure in the end.

At even larger densities where baryon hard cores overlap, $\rho \sim r_{\text {hard }}^{-3}$, most clusters get quantum percolated, and delocalized quark states become more populated as shown in the far right panel of Fig. 12. In this way the quark Fermi sea grows up and develops with low momentum states of quarks saturated. Isolated baryon clusters would become fewer, although they should still remain due to quantum interference effects. Possibly, therefore, $f_{\text {quark }}(k)$ sustains contributions from localized (interpreted as confined) states, as sketched by the red area in $f_{\text {quark }}(k)$ in the far right panel of Fig. 12. These localized states on top of the Fermi sea may be regarded as relativistic baryons with $N_{\mathrm{c}}$-quarks collectively moving in the same direction. This is in contrast to the case without the Fermi sea, where moving directions of $N_{\mathrm{c}}$-quarks are not aligned, leaving a small baryon momentum. Changes from the nonrelativistic to relativistic regime can be one of sources for stiffening in equations of state $[29,30]$.

The modeling of dense matter as a superposition of localized and delocalized quark wave functions may be relevant to physical observables involving excited modes rather than bulk thermodynamics. Thus, the effects on the neutron star EoS may be limited, but the transport coefficients such as the heat conductivity, the baryon number diffusion constant, the viscosity, and so on could be sensitive to details of mode-by-mode localization/delocalization. Another quantity sensitive to excitation modes is the entropy density for which nuclear matter and quark matter contribute in parametrically distinct ways. The soft deconfinement contains both of these contributions as in Fig. 12, and it may be possible to define an effective critical density separating nuclear, soft deconfinement, and hard deconfinement regimes, in a way similar to the pseudocritical temperature in finite temperature QCD. In principle these effects can be studied from phenomenology such as protoneutron stars and neutron star mergers. The photo production rate may be also an interesting indicator in a similar fashion to high- $T$ matter coupled with the Polyakov loop [70].

\section{CONCLUSIONS}

In this work we proposed two characterizations of quark deconfinement, namely, hard deconfinement and soft deconfinement. It is conceptually straightforward to understand hard deconfinement along the lines of a conventional picture of classical percolation of baryons. Once the 
nucleon core regions overlap, thermodynamic properties are dominated by the energy-momentum tensor in the nucleon core that could be available by measuring the gravitational form factors of the nucleon in deeply virtual Compton scattering. Based on this speculation, we quantified the internal structures of a nucleon using a chiral soliton model, and estimated the equation-of-state of its compact core. We found that the nucleon EoS obtained in this way is fairly consistent with the empirical EoSs known from neutron star phenomenology. We also discussed implications of partial chiral symmetry restoration in dense matter which lead to a stiffer EoS, a direction bringing the nucleon core EoS closer to other empirical EoSs.

Soft deconfinement is a more subtle notion. Microscopically, expectation values are taken over ensembles of various quantum states. Consider various snapshots of baryon configurations. As the baryon density increases, the cluster size in such snapshots of baryon configurations becomes larger. Then, eventually, quark wave functions are more and more delocalized and the quantum percolation of quarks occurs from smaller momentum modes of quarks.

The appropriate physical interpretation of soft deconfinement should thus be based on the localization or delocalization of quark states. Even if they are localized, quark wave functions may extend over a wider range than a single nucleon, and so there is no sharp identification of confinement/deconfinement. Such gradual changes of the delocalization range would give us detailed insights on the quark-hadron continuity, and at the same time, useful clues to resolve intuitive views of quarkyonic matter and its field-theoretical modeling (see also Ref. [71] for a recent attempt). In contrast to hard deconfinement for which we presented quantitative estimates, our discussions on soft deconfinement are limited to a qualitative level. It is a very interesting and challenging problem to formulate a quantitative description of $\rho(E)$ and reveal its gauge (in) dependence, so that the mobility edge trajectory can be drawn and the detailed momentum shell structures could be clarified.

\section{ACKNOWLEDGMENTS}

We thank Gordon Baym, Yuki Fujimoto, Hideaki Iida, Giorgio Torrieri, Naoki Yamamoto for useful discussions and comments. K. F. was supported by Japan Society for the Promotion of Science (JSPS) KAKENHI Grants No. 18H01211 and No. 19K21874. T. K. was supported by NSFC Grant No. 11875144 .
[1] N. Itoh, Prog. Theor. Phys. 44, 291 (1970).

[2] J. C. Collins and M. Perry, Phys. Rev. Lett. 34, 1353 (1975).

[3] K. Fukushima and T. Hatsuda, Rep. Prog. Phys. 74, 014001 (2011).

[4] K. Fukushima and C. Sasaki, Prog. Part. Nucl. Phys. 72, 99 (2013).

[5] M. Buballa and S. Carignano, Prog. Part. Nucl. Phys. 81, 39 (2015).

[6] C. S. Fischer, Prog. Part. Nucl. Phys. 105, 1 (2019).

[7] X. Luo and N. Xu, Nucl. Sci. Tech. 28, 112 (2017).

[8] S. Muroya, A. Nakamura, C. Nonaka, and T. Takaishi, Prog. Theor. Phys. 110, 615 (2003).

[9] G. Aarts, J. Phys. Conf. Ser. 706, 022004 (2016).

[10] M. Asakawa, U. W. Heinz, and B. Muller, Phys. Rev. Lett. 85, 2072 (2000).

[11] S. Jeon and V. Koch, Phys. Rev. Lett. 85, 2076 (2000).

[12] S. Ejiri, F. Karsch, and K. Redlich, Phys. Lett. B 633, 275 (2006).

[13] P. Braun-Munzinger, J. Stachel, J. Wessels, and N. Xu, Phys. Lett. B 344, 43 (1995).

[14] J. Cleymans, D. Elliott, H. Satz, and R. Thews, Z. Phys. C 74, 319 (1997).

[15] J. Cleymans and K. Redlich, Phys. Rev. C 60, 054908 (1999).

[16] P. Braun-Munzinger, D. Magestro, K. Redlich, and J. Stachel, Phys. Lett. B 518, 41 (2001).
[17] F. Becattini, J. Manninen, and M. Gazdzicki, Phys. Rev. C 73, 044905 (2006).

[18] A. Andronic, P. Braun-Munzinger, and J. Stachel, Nucl. Phys. A772, 167 (2006).

[19] N. Cabibbo and G. Parisi, Phys. Lett. 59B, 67 (1975).

[20] A. Andronic et al., Nucl. Phys. A837, 65 (2010).

[21] T. D. Cohen and V. Krejcirik, J. High Energy Phys. 08 (2011) 138.

[22] A. Andronic, P. Braun-Munzinger, J. Stachel, and M. Winn, Phys. Lett. B 718, 80 (2012).

[23] H. Heiselberg, Phys. Rev. Lett. 72, 3013 (1994).

[24] P. B. Arnold, D. T. Son, and L. G. Yaffe, Phys. Rev. D 59, 105020 (1999).

[25] E. Witten, Nucl. Phys. B160, 57 (1979).

[26] L. McLerran and R. D. Pisarski, Nucl. Phys. A796, 83 (2007).

[27] G. Baym, T. Hatsuda, T. Kojo, P. D. Powell, Y. Song, and T. Takatsuka, Rep. Prog. Phys. 81, 056902 (2018).

[28] C. M. Soukoulis, Q. Li, and G. S. Grest, Phys. Rev. B 45, 7724 (1992).

[29] L. McLerran and S. Reddy, Phys. Rev. Lett. 122, 122701 (2019).

[30] K. S. Jeong, L. McLerran, and S. Sen, Phys. Rev. C 101, 035201 (2020).

[31] W. Xiong et al., Nature (London) 575, 147 (2019).

[32] A. Filin, V. Baru, E. Epelbaum, H. Krebs, D. Möller, and P. Reinert, Phys. Rev. Lett. 124, 082501 (2020). 
[33] N. Kaiser and E. Passemar, Eur. Phys. J. A 55, 16 (2019).

[34] J. Gasser, H. Leutwyler, and M. Sainio, Phys. Lett. B 253, 252 (1991).

[35] J. Gasser, H. Leutwyler, and M. Sainio, Phys. Lett. B 253, 260 (1991).

[36] P. Schweitzer, Phys. Rev. D 69, 034003 (2004).

[37] M. Hoferichter, J. Ruiz de Elvira, B. Kubis, and U.-G. Meißner, Phys. Rev. Lett. 115, 192301 (2015).

[38] G. Brown, M. Rho, and W. Weise, Nucl. Phys. A454, 669 (1986).

[39] A. W. Thomas and W. Weise, The Structure of the Nucleon (Wiley, Berlin, 2001).

[40] U. G. Meissner, N. Kaiser, A. Wirzba, and W. Weise, Phys. Rev. Lett. 57, 1676 (1986).

[41] U. Meissner, N. Kaiser, and W. Weise, Nucl. Phys. A466, 685 (1987).

[42] G. S. Adkins and C. R. Nappi, Phys. Lett. 137B, 251 (1984).

[43] Y. Igarashi, M. Johmura, A. Kobayashi, H. Otsu, T. Sato, and S. Sawada, Nucl. Phys. B259, 721 (1985).

[44] K. Kawarabayashi and M. Suzuki, Phys. Rev. Lett. 16, 384 (1966).

[45] Riazuddin and Fayyazuddin, Phys. Rev. 147, 1071 (1966).

[46] C. Cebulla, K. Goeke, J. Ossmann, and P. Schweitzer, Nucl. Phys. A794, 87 (2007).

[47] K. Goeke, J. Grabis, J. Ossmann, M. Polyakov, P. Schweitzer, A. Silva, and D. Urbano, Phys. Rev. D 75, 094021 (2007).

[48] V. Burkert, L. Elouadrhiri, and F. Girod, Nature (London) 557, 396 (2018).

[49] M. V. Polyakov and P. Schweitzer, Int. J. Mod. Phys. A 33, 1830025 (2018).

[50] K. Hebeler, J. M. Lattimer, C. J. Pethick, and A. Schwenk, Astrophys. J. 773, 11 (2013).

[51] F. Douchin and P. Haensel, Astron. Astrophys. 380, 151 (2001).
[52] Y. Fujimoto, K. Fukushima, and K. Murase, Phys. Rev. D 101, 054016 (2020).

[53] M. Drews and W. Weise, Phys. Rev. C 91, 035802 (2015).

[54] B. Friman and W. Weise, Phys. Rev. C 100, 065807 (2019).

[55] A. Rajan, T. Gorda, S. Liuti, and K. Yagi, Proc. Sci., LATTICE2018 (2018) 180 [arXiv:1810.01130].

[56] F. Karsch and H. Satz, Phys. Rev. D 21, 1168 (1980).

[57] P. Castorina, K. Redlich, and H. Satz, Eur. Phys. J. C 59, 67 (2009).

[58] S. Lottini and G. Torrieri, Phys. Rev. Lett. 107, 152301 (2011).

[59] G. Baym, T. Hatsuda, M. Tachibana, and N. Yamamoto, J. Phys. G 35, 104021 (2008).

[60] N. Kaiser, Eur. Phys. J. A 48, 135 (2012).

[61] S. Torquato and Y. Jiao, J. Chem. Phys. 137, 074106 (2012); 141, 159901 (2014).

[62] B. K. Chakrabarti, K. K. Bardhan, and A. K. Sen, Quantum and Semi-classical Percolation and Breakdown in Disordered Solids, Lecture Notes in Physics (Springer, Berlin, Heidelberg, 2009).

[63] S. Kirkpatrick and T. P. Eggarter, Phys. Rev. B 6, 3598 (1972).

[64] T. Mano and T. Ohtsuki, J. Phys. Soc. Jpn. 86, 113704 (2017).

[65] C. M. Soukoulis, E. N. Economou, and G. S. Grest, Phys. Rev. B 36, 8649 (1987).

[66] A. M. Garcia-Garcia and J. C. Osborn, Nucl. Phys. A770, 141 (2006).

[67] M. Giordano, T. G. Kovacs, and F. Pittler, Phys. Rev. D 95, 074503 (2017).

[68] L. Holicki, E.-M. Ilgenfritz, and L. von Smekal, Proc. Sci., LATTICE2018 (2018) 180.

[69] T. Maruyama, K. Niita, K. Oyamatsu, T. Maruyama, S. Chiba, and A. Iwamoto, Phys. Rev. C 57, 655 (1998).

[70] D. Satow and W. Weise, Phys. Rev. D 92, 056001 (2015).

[71] G. Cao and J. Liao, arXiv:2007.02028. 